\title{
Risky Decisions in the Large and in the Small: Theory and Experiment
}

\author{
By James C. Cox and Vjollca Sadiraj
}

\section{INTRODUCTION}

Much of the literature on theories of decision making under risk has emphasized differences between theories. One enduring theme has been the attempt to develop a distinction between "normative" and "descriptive" theories of choice. Bernoulli (1738) introduced log utility because expected value theory was alleged to have descriptively incorrect predictions for behavior in St. Petersburg games. Much later, Kahnemann and Tversky (1979) introduced prospect theory because of the alleged descriptive failure of expected utility theory (von Neumann and Morgenstern 1947).

In this essay, we adopt a different approach. Rather than emphasizing differences between theories of decision making under risk, we focus on their similarities - and on their common problems when viewed as "positive" (that is, testable) theories. We examine five prominent theories of decision making under risk - expected value theory, expected utility theory, cumulative prospect theory, rank dependent utility theory, and dual theory of expected utility - and explain the fundamental problems inherent in all of them.

We focus on two generic types of problems that are common to theories of risky decisions: (a) generalized St. Petersburg paradoxes; and (b) implications of implausible risk aversion. We also discuss the recent generalization of the risk aversion calibration literature, away from its previously exclusive focus on implications of decreasing marginal utility of money, to include implications of probability transformations (Cox et al. 2007). We also note that much recent discussion of alleged "behavioral" implications of Rabin's (2000) concavity calibration proposition has not involved any credible observations of behavior, and discuss possible remedies 
including the experiments reported in Cox et al. (2007) and other designs for experiments outlined below.

The second section in the chapter discusses "utility functionals" that represent risk preferences for the five representative theories of decision making under risk listed above and defines a general class of theories that contains all of them. In section 3, we discuss issues that arise if the domain on which theories of decision making under risk aversion are defined is unbounded, as in the seminal papers on the expected utility theory of risk aversion by Arrow (1971) and Pratt (1964) and the textbook by Laffont (1989). These prominent developments of the theory assume bounded utility (see, for example, Arrow 1971, p. 92 and Laffont 1989, p. 8) in order to avoid generalized St. Petersburg paradoxes on an unbounded domain. We demonstrate that this traditional assumption of bounded utility substitutes one type of problem for another because, on unbounded domains, bounded utility implies implausible risk aversion (as defined in section 3.1 below).

Our discussion is not confined to expected utility theory. We demonstrate that, on an unbounded domain, all five of the prominent theories of risky decisions have arguably implausible implications: with unbounded utility (or "value" of "money transformation") functions there are generalized St. Petersburg paradoxes and with bounded utility functions there are implausible aversions to risk taking.

One possible reaction to the analysis in section 3 might be: "So what? All empirical applications of risky decision theory are on bounded domains, so why should an applied economist care about any of this?" The answer to this question is provided in subsequent sections of the chapter in which we elucidate how the analysis on an unbounded domain causes one to ask new questions about applications of risky decision theories on bounded domains. We explain how finite St. Petersburg games provide robustness tests for empirical work on risk aversion on bounded domains. We discuss parametric forms of money transformation (or utility) functions commonly used in econometric analysis of lottery choice data and calibrate implications of 
parameter estimates in the literature for binary lottery preferences. These implied preferences over binary lotteries provide the basis for robustness tests of whether the reported parameter estimates can, indeed, rationalize the risk preferences of the subjects.

Finally, we consider risk aversion patterns that are not based on parametric forms of money transformation functions or probability transformation functions. We summarize recent within-subjects experiments on the empirical validity of the postulated patterns of risk aversion underlying the concavity calibration literature and extensions of this literature to include convexity calibration of probability transformations. We also explain why some across-subjects experiments on concavity calibration reported in the literature do not, in fact, have any implications for empirical validity of calibrated patterns of small stakes risk aversion.

\section{REPRESENTATIVE THEORIES OF DECISION UNDER RISK}

Let $\left\{Y_{n}, P_{n}\right\}$ denote a lottery that pays amounts of money $Y_{n}=\left[y_{n}, y_{n-1}, \cdots, y_{1}\right]$ with respective probabilities $P_{n}=\left[p_{n}, p_{n-1}, \cdots, p_{1}\right], n \in N$, the set of integers, and $y_{j} \geq y_{j-1}, p_{j} \geq 0$, for $j=1,2, \cdots, n$, and $\sum_{j=1}^{n} p_{j}=1$. This essay is concerned with theories of preferences over such lotteries. In representing the theories with utility functions, it will be useful to also define notation for the probabilities of all outcomes except $y_{j}: P_{n}^{-j}=\left[p_{n}, p_{n-1}, \cdots p_{j-1}, p_{j+1}, \cdots, p_{1}\right]$.

We discuss expected value theory (Bernoulli 1738), expected utility theory (von Neumann and Morgenstern 1947), dual theory of expected utility (Yaari 1987), rank dependent utility theory (Quiggin 1982, 1993), and cumulative prospect theory (Tversky and Kahneman 1992). All five of these theories represent risk preferences with utility functionals that have a common form that is additive across states of the world (represented by the index $j=1,2, \cdots n$ ). This additive form defines a class 招 of decision theories that contains the above five prominent 
theories. We will review utility functionals for these five theories before stating the general functional form that can represent each theory's typical functional as a special case.

Expected value theory represents preferences over the lotteries with a functional of the form

$$
U_{E V}\left(\left\{Y_{n}, P_{n}\right\}\right)=a+b \sum_{j=1}^{n} p_{j} y_{j}, b>0
$$

The same EV preferences are represented when functional (1) is simplified by setting $a=0$ and $b=1{ }^{1}$ We will avoid some otherwise tedious repetition by using similar affine transformations of utility (or "money transformation") functions, without explicit discussion, for other theories considered in subsequent paragraphs.

Expected utility theory represents preferences over the lotteries with a functional that can be written as

$$
U_{E U}\left(\left\{Y_{n}, P_{n}\right\}\right)=\sum_{j=1}^{n} p_{j} u\left(y_{j}, w\right)
$$

where $w$ is the agent's initial wealth. Utility functionals (1) and (2) are both linear in probabilities, which in the case of expected utility theory is an implication of the independence axiom. Functional (2) is linear in money payoffs $y$ only if the agent is risk neutral. Expected utility theory contains (at least) three models. The expected utility of terminal wealth model (Pratt 1964, Arrow 1971) assumes that risk preferences are defined over terminal wealth, i.e. that the "money transformation function" (or utility function) $u$ takes the form $u(y, w)=\varphi_{E U W}(y+w)$. The expected utility of income model commonly used in bidding theory assumes that risk preferences are independent of wealth, i.e. that the money transformation function takes the form

\footnotetext{
${ }^{1}$ The EV theory of risk preferences has the same implications if terminal wealth rather than income is assumed to be the random lottery payoff in the functional in statement (1).
} 
$u(y, w)=\varphi_{E U I}(y) .^{2}$ The expected utility of initial wealth and income model (Cox and Sadiraj 2006) represents risk preferences with a money transformation function of the ordered pair of arguments $(y, w)$. This model includes as special cases the terminal wealth model in which there is full asset integration, the income model in which there is no asset integration, and other models in which there is partial asset integration. ${ }^{3}$

The dual theory of expected utility represents preferences over the lotteries with a functional of the form

$$
U_{D U}\left(\left\{Y_{n}, P_{n}\right\}\right)=\sum_{j=1}^{n}\left[f\left(\sum_{k=j}^{n} p_{k}\right)-f\left(\sum_{k=j+1}^{n} p_{k}\right)\right] \cdot y_{j}
$$

Functional (3) is linear in payoffs as a consequence of the dual independence axiom. The transformation function $f$ for decumulative probabilities is strictly convex if the agent is risk averse. If the agent is risk neutral then the decumulative probability transformation function $f$ is linear and hence the utility functional (3) is linear in probabilities (in that special case).

Rank dependent utility theory represents preferences over the lotteries with a functional of the form ${ }^{4}$

$$
U_{R D}\left(\left\{Y_{n}, P_{n}\right\}\right)=\sum_{j=1}^{n}\left[q\left(\sum_{k=1}^{j} p_{k}\right)-q\left(\sum_{k=1}^{j-1} p_{k}\right)\right] \cdot \mu\left(y_{j}\right) .
$$

Prospect theory transforms both probabilities and payoffs differently for losses than for gains. In the original version of cumulative prospect theory, Tversky and Kahneman (1992) defined gains and losses in a straightforward way relative to zero income. Some more recent versions of the theory have reintroduced the context-dependent gain/loss reference points used in

\footnotetext{
2 The expected utility of income model was used to develop much of Bayesian-Nash equilibrium bidding theory. See, for examples: Holt (1980), Harris and Raviv (1981), Riley and Samuelson (1981), Cox et al. (1982), Milgrom and Weber (1982), Matthews (1983), Maskin and Riley (1984), and Moore (1984).

${ }^{3}$ See Harrison, et al. (2007b) and Heinemann (2008) for empirical applications of partial asset integration models.

${ }^{4}$ We write the functional for rank dependent utility theory with transformation of cumulative probabilities in the same way as Quiggin $(1982,1993)$. Some later expositions of this theory use a logically equivalent representation with transformation of decumulative probabilities.
} 
the original version of "non-cumulative" prospect theory (Kahneman and Tversky 1979). Let $r$ be the possibly non-zero reference point value of money payoffs that determines which payoffs are "losses" $(y<r)$ and which payoffs are "gains" $(y>r)$. And let the lottery money payoffs $y_{j}$ be less than $r$ for $j \leq N_{r}$. Then risk preferences for cumulative prospect theory can be represented with a functional of the form

$$
\begin{aligned}
U_{C P}\left(\left\{Y_{n}, P_{n}\right\}\right)= & \sum_{j=1}^{N_{r}}\left[w^{-}\left(\sum_{k=1}^{j} p_{k}\right)-w^{-}\left(\sum_{k=1}^{j-1} p_{k}\right)\right] \cdot v^{-}\left(y_{j}-r\right) \\
& +\sum_{j=N_{r}+1}^{n}\left[w^{+}\left(\sum_{k=j}^{n} p_{k}\right)-w^{+}\left(\sum_{k=j+1}^{n} p_{k}\right)\right] \cdot v^{+}\left(y_{j}-r\right) .
\end{aligned}
$$

In utility functional (5): $v^{-}$is the value function for losses; $v^{+}$is the value function for gains; and $w^{-}$and $w^{+}$are the corresponding weighting functions for probabilities (or "capacities"). There is a discontinuity in the slope of the value function at payoff equal to the reference payoff $r$, which is "loss aversion." 5 A strictly concave value function for gains $v^{+}$and associated Sshaped probability weighting function $w^{+}$are commonly used in applications of prospect theory.

The analysis in subsequent sections will use a general form of utility functional that, with suitable interpretations, represents all of the above theories of decision making under risk. Let $h_{D}$ be a probability transformation function for theory D. Let a positively monotonic function $\varphi_{D}$ denote a money transformation function for theory $D$. Let $w$ be the amount of initial wealth. Let 政 be the set of decision theories $D$ that represent preferences over lotteries by utility functionals with the form:

$$
U_{D}\left(\left\{Y_{n}, P_{n}\right\}\right)=\sum_{j=1}^{n} h_{D}\left(p_{j}, P_{n}^{-j}\right) \varphi_{D}\left(y_{j}, w\right)
$$

\footnotetext{
${ }^{5}$ Loss aversion, when defined as a discontinuity in the slope of the utility function at zero income, is
} consistent with the expected utility of income model (Cox and Sadiraj 2006). 
The additive-across-states form of (6) defines the class $\mathbb{\$}$ of theories we discuss. This class contains all of the popular examples of theories discussed above. Many results in following sections apply to all theories in class 政. Discussion in subsequent sections will describe some instances in which specific differences between the utility functionals for distinct theories are relevant to the analysis of properties of the theories we examine.

Before proceeding to analyze the implications of functionals of form (6), it might be helpful to further discuss interpretations of (6) using the examples of theories D in $\mathbb{\text { I }}$ mentioned above. In the case of expected value theory, the probability transformation function $h_{D}$ in (6), written as $h_{E V}$, is a constant function of $P_{n}^{-j}$ and is the identity map of $p_{j}: h_{E V}\left(p_{j}, P_{n}^{-j}\right)=p_{j}$ for all $\left(p_{j}, P_{n}^{-j}\right)$. The money transformation function $\varphi_{E V}$ is linear in $y($ or in $y+w)$.

Functional (6) is interpreted for expected utility theory as follows. The probability transformation function $h_{E U}$ is a constant function of $P_{n}^{-j}$ and is the identity map of $p_{j}$, as a consequence of the independence axiom. Interpretations of the money transformation function $\varphi_{E U}$ vary across three expected utility models, as explained above.

The interpretation of functional (6) for the dual theory of expected utility is as follows. The money transformation function $\varphi_{D U}$ is always linear in $y$ (or in $y+w$ ) as a consequence of the dual independence axiom. The probability transformation function $h_{D U}$ is a composition of functions of $\sum_{k \geq j} p_{k}$ and $\sum_{k \geq j+1} p_{k}$ as shown in statement (3). The probability transformation function is linear only if the agent is risk neutral.

Functional (6) is interpreted for rank dependent utility theory as follows. The money transformation function $\varphi_{R D}$ is a constant function of $w$ and is increasing in $y$. The probability 
transformation function $h_{R D}$ is a composition of functions of $\sum_{k \leq j} p_{k}$ and $\sum_{k \leq j-1} p_{k}$ as shown in statement (4).

The interpretation of functional (6) for cumulative prospect theory is the most complicated one because of the various interdependent special features of that theory. The money transformation function $\varphi_{C P}$ is a constant function of $w$ and increasing in $y$, with a discontinuous change in slope at $y=r$; furthermore, in some versions of the theory the reference point income $r$ can be variable and context dependent. As shown in (5), the probability transformation function $h_{C P}$ is a composition of functions of $\sum_{k=1}^{j} p_{k}$ and $\sum_{k=1}^{j-1} p_{k}$, when $y<r$, and a composition of functions of $\sum_{k=j}^{n} p_{k}$ and $\sum_{k=j+1}^{n} p_{k}$, when $y \geq r$.

We now proceed to derive some implications of theories in class $\mathbb{\text { I }}$ that have prefences over lotteries that can be represented by utility functionals with the form given by statement (6).

\section{THEORY FOR UNBOUNDED DOMAIN:}

\section{ST. PETERSBURG PARADOX OR IMPLAUSIBLE RISK AVERSION}

We here discuss theories of decision making under risk in the domain of discourse adopted in classic expositions of expected utility theory such as Arrow (1971) and Pratt (1964), as well as in advanced textbook treatments such as Laffont (1989). In contrast to those studies, our discussion is not confined to expected utility theory but, instead, applies to all decision theories in class $\mathbb{\text { 相. }}$

For any money transformation function $\varphi_{D}$, defined on an unbounded domain, there can be only two exclusive cases: the function is either unbounded from above or bounded. In this section we consider both of these two cases and show that all decision theories in class $\mathbb{\text { II }}$ have

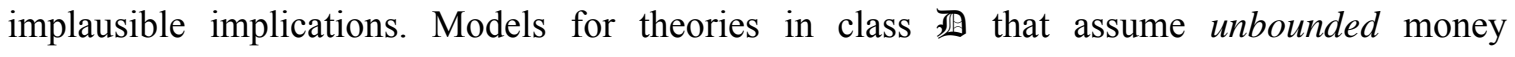
transformation functions are characterized by generalized St. Petersburg paradoxes. Models for 
theories in class $\mathbb{\text { Th }}$ that assume bounded money transformation functions are characterized by implausible risk aversion, as defined below.

\subsection{Unbounded Money Transformation Functions}

Some examples of unbounded money transformation functions are linear functions, power functions, and logarithmic functions. Daniel Bernoulli (1738) introduced the St. Petersburg paradox (as described in the next paragraph) that questioned the plausibility of expected value theory. Bernoulli offered log utility of money as a solution to the St. Petersburg paradox that preserves linearity in probabilities (and in that way anticipated subsequent development of expected utility theory). However, unbounded monotonic money transformation functions (including log functions) do not eliminate generalized St. Petersburg paradox problems for expected utility theory (Arrow 1971, p. 92; Samuelson 1977). We here explain that unbounded money transformation functions produce similar plausibility problems for other decision theories in class 政 (see also Rieger and Wang 2006).

The original St. Petersburg game pays $2^{k}$ when a fair coin comes up heads for the first time on flip $k$, an event with probability $1 / 2^{k}$. The game can be represented by $\left\{Y_{\infty}, P_{\infty}\right\}=$ $\left\{2_{\infty}, 1 / 2_{\infty}\right\}$ where $2_{\infty}=\left[\cdots, 2^{n}, 2^{n-1}, \cdots, 2\right]$ and $1 / 2_{\infty}=\left[\cdots, 1 / 2^{n}, 1 / 2^{n-1}, \cdots, 1 / 2\right]$. Expected value theory evaluates this lottery according to $U_{E V}\left(\left\{2_{\infty}, 1 / 2_{\infty}\right\}\right)=\sum_{k=1}^{\infty} 2^{k} \times\left(1 / 2^{k}\right)=\infty$. Bernoulli (1738) famously reported that most people stated they would be unwilling to pay more than a small finite amount to play this game. A log utility of money function, offered by Bernoulli as an alternative to the linear utility of money function, does solve the paradox of the original St. Petersburg lottery because $\sum_{k=1}^{\infty}\left[\ln \left(2^{k}\right) \times\left(1 / 2^{k}\right)\right]=2 \ln (2)$ is finite. 
It is now well known that the log utility of money function cannot solve the paradox of a slightly modified version of the original St. Petersburg game: pay $\exp \left(2^{k}\right)$ when a fair coin comes up heads for the first time on flip $k$. The problem is not with the log function per se. No unbounded money transformation function can eliminate problems of the St. Petersburg type of paradox for expected utility theory. For any $\varphi_{E U}$ not bounded from above, define a sequence of payments $X_{E U}=\left\{x_{n}: n \in N\right\}$ such that, for all $j, \varphi_{E U}\left(z_{k}\right) \geq 2^{k}$, where $z_{k}$ equals either $x_{k}$ or $w+x_{k}$ depending on whether one is applying the expected utility of income model or the expected utility of terminal wealth model. ${ }^{6}$ The expected utility of a St. Petersburg game that pays $x_{k}$ (instead of $2^{k}$ ) when a fair coin comes up heads for the first time on flip $k$ is infinite. This is shown for the expected utility of income model by

$$
U_{E U I}\left(\left\{x_{\infty}, 1 / 2_{\infty}\right\}\right)=\sum_{k=1}^{\infty}\left(\frac{1}{2}\right)^{k} \varphi_{E U I}\left(x_{k}\right) \geq \sum_{k=1}^{\infty}\left(\frac{1}{2}\right)^{k} 2^{k}=\infty
$$

Hence an expected utility maximizer whose preferences are represented with money transformation function $\varphi_{E U}$ for amounts of income would prefer game $X_{E U}$ to any certain amount of money, no matter how large. Similarly, an expected utility maximizer whose preferences are represented with money transformation function $\varphi_{E U}$ of amounts of terminal wealth would be willing to pay any amount $\pi$ up to his entire (finite) amount of initial wealth $w$ to play game $X_{E U}$ since, for all $\pi \leq w$,

$$
U_{E U W}\left(\left\{x_{\infty}, 1 / 2_{\infty}\right\}\right)=\sum_{k=1}^{\infty}\left(\frac{1}{2}\right)^{k} \varphi_{E U W}\left(w-\pi+x_{k}\right) \geq \sum_{k=1}^{\infty}\left(\frac{1}{2}\right)^{k} 2^{k}=\infty>\varphi_{E U W}(w) .
$$

\footnotetext{
${ }^{6}$ If there exists an inverse function $\varphi_{E U}^{-1}$ then the sequence of payoffs $Z_{n}=\varphi_{E U}^{-1}\left(2^{n}\right)$ provides a generalized St. Petersburg game with infinite expected utility.
} 
The following proposition generalizes this result and demonstrates that unbounded money transformation functions produce similar plausibility problems for all decision theories in class 西. One has:

Proposition 1. Let an agent's preferences defined on an unbounded domain be represented by functional (6) with an unbounded money transformation function $\varphi$ and a strictly positive probability transformation function $h$. The agent will reject any finite amount of money in favor of a St. Petersburg lottery that pays $x_{k} \in X_{\varphi, h}=\left\{x_{j} \mid j \in N, \varphi\left(x_{j}\right) \geq 1 / h\left(1 / 2^{j},\left[1 / 2_{\infty}\right]^{-j}\right)\right\}$ when a fair coin comes up heads for the first time on flip $k$.

Proof: Apply Lemma A.1 in appendix A.1.

To illustrate Proposition 1, we report examples of generalized St. Petersburg games for some of the alternatives to expected utility theory in class $\mathbb{\rrbracket}$, including dual theory of expected utility, rank dependent utility theory, and cumulative prospect theory.

First consider the dual theory of expected utility with positively monotonic and convex transformation $f$ for decumulative probabilities. According to this theory, the St. Petersburg game that pays $x_{n}$ if the first head appears on flip $n$ is evaluated by

$$
U_{D U}\left(X_{D U}\right)=\sum_{n, X_{n} \in X_{D U}} x_{n}\left(f\left(\sum_{k=n}^{\infty} \frac{1}{2^{k}}\right)-f\left(\sum_{k=n+1}^{\infty} \frac{1}{2^{k}}\right)\right)=\sum_{n, x_{n} \in X_{D U}} x_{n}\left(f\left(2^{1-n}\right)-f\left(2^{-n}\right)\right)
$$

which is unbounded from above for $x_{n}$ from $X_{D U}=\left\{x_{n}: n \in N, x_{n} \geq 1 /\left[f\left(2^{1-n}\right)-f\left(2^{-n}\right)\right]\right\}$.

Next, consider rank dependent utility theory with transformation function $q$ (for cumulative probabilities). Since $\varphi_{R D}$ is not bounded from above, one can find a sequence of payments $X_{R D}=\left\{x_{n}: n \in N, \varphi_{R D}\left(x_{n}\right) \geq 1 /\left[q\left(1-2^{-n}\right)-q\left(1-2^{-(n-1)}\right)\right\}\right.$. The rank dependent utility of the St. Petersburg game that pays $x_{n}, x_{n} \in X_{R D}$ if a fair coin comes up heads for the first time on flip $n$ is 


$$
U_{R D}\left(X_{R D}\right)=\sum_{n=1}^{\infty} \varphi_{R D}\left(x_{j}\right)\left(q\left(\sum_{k=1}^{n} \frac{1}{2^{k}}\right)-q\left(\sum_{k=1}^{n-1} \frac{1}{2^{k}}\right)\right)=\sum_{n=1}^{\infty} \varphi_{R D}\left(x_{n}\right)\left(q\left(1-2^{-n}\right)-q\left(1-2^{-(n-1)}\right)\right)
$$

which is unbounded by construction of $X_{R D}$.

Finally, consider cumulative prospect theory with reference point equal to a given amount of money $r$. Let $\varphi_{C P}^{-}$be the money transformation (or "value") function for losses and $\varphi_{C P}^{+}$be the money transformation function for gains. Let $w^{-}$be the probability transformation in the loss domain and $w^{+}$be the probability transformation function in the gain domain. Assume loss aversion: a discontinuity of the slope of the value function at $x=r$. Define $X_{C P}=\left\{x_{n}: n \in N, \varphi_{C P}^{+}\left(x_{n}-r\right) \geq 1 /\left[w^{+}\left(\sum_{k \geq n} 2^{-k}\right)-w^{+}\left(\sum_{k \geq n+1} 2^{-k}\right)\right]\right\}$. Without loss of generality, let $r$ be between $x_{j}$ and $x_{j+1}$, for some $j \in N$. The St. Petersburg game that pays $x_{n} \in X_{C P}$ if a fair coin comes up heads for the first time on flip $n$ is evaluated by cumulative prospect theory as follows:

$$
\begin{aligned}
U_{C P}\left(X_{C P}\right) & =\sum_{i=1}^{j} \varphi_{P T}^{-}\left(x_{i}-r\right)\left(w^{-}\left(\sum_{k=1}^{i} \frac{1}{2^{k}}\right)-w^{-}\left(\sum_{k=1}^{i-1} \frac{1}{2^{k}}\right)\right) \\
& +\sum_{n=j+1}^{\infty} \varphi_{P T}^{+}\left(x_{n}-r\right)\left(w^{+}\left(\sum_{k=n}^{\infty} \frac{1}{2^{k}}\right)-w^{+}\left(\sum_{k=n+1}^{\infty} \frac{1}{2^{k}}\right)\right)
\end{aligned}
$$

Note that $U_{C P}\left(X_{C P}\right)$ is unbounded from above since the first term on the right hand side is always finite whereas the second term on the right is unbounded from above by construction of $X_{C P}$. All of the above, of course, is also true if the reference point $r$ is set equal to zero; therefore a prospect theory agent would prefer the lottery $X_{C P}$ to any finite amount of money.

In this way, for any unbounded money transformation function one can construct a generalized St. Petersburg paradox for any of the five decision theories when they are defined on an unbounded domain. Bounded money transformation functions are immune to critique with 
generalized St. Petersburg lotteries. We will explain, however, that on unbounded domains bounded money transformation functions imply implausible risk aversion, as next defined.

Let $\left\{y_{2}, p ; y_{1}\right\}$ denote a binary lottery that pays the larger amount $y_{2}$ with probability $p$ and the smaller amount $y_{1}$ with probability $1-p$. We define "implausible risk aversion" for binary lotteries as follows.

(I*) Implausible risk aversion: for any $z$ there exists a finite $L$ such that the certain amount of money $z+L$ is preferred to the lottery $\{\infty, 0.5 ; z\}$.

\subsection{Bounded Money Transformation Functions}

In order to escape the behaviorally implausible implications of the generalized St. Petersburg paradox for any theory in class 政 defined on an unbounded domain, one needs to use a money transformation function that is bounded from above. But bounded money transformation functions imply implausible risk aversion, as we shall explain. We start with two illustrative examples using bounded, parametric money transformation functions commonly used in the literature. Subsequently, we present a general proposition for bounded money transformation functions that applies to all theories in class 㨁.

One of the commonly used money transformation (or utility) functions in the literature is the (concave transformation of the) exponential function, commonly known as CARA, defined as: ${ }^{7}$

$$
\varphi_{D}(y)=\left(1-\mathrm{e}^{-\lambda y}\right), \lambda>0
$$

Define $g_{D}(0.5) \equiv h_{D}(0.5,[0.5])$ as the transformed probability of the higher outcome in a binary lottery with 0.5 probabilities of the two payoffs. For the exponential money transformation function in statement (13), it can be easily verified that decision theory D implies that a certain

\footnotetext{
${ }^{7}$ In the context of the expected utility of terminal wealth model, utility function (13) represents constant absolute risk averse preferences, which is the source of the name CARA.
} 
payoff in amount $x+\ln \left(1-g_{D}(0.5)\right)^{-1 / \lambda}$ is preferred to $\{\infty, 0.5 ; x\}$, for all $x$. For example, an expected utility maximizing agent (for whom $g(0.5)=0.5$ ) with $\lambda=0.29$ would prefer a certain payoff of $\$ 25$ (or, in the terminology of Proposition $2, x+L=\$ 22+\$ 3$ ) to the lottery $\{\$ \infty, 0.5 ; \$ 22\}$. The parameter value $\lambda=0.07$ implies that an expected utility maximizing agent would prefer $\$ 32$ for sure to the lottery $\{\$ \infty, 0.5 ; \$ 22\}$.

Another common parametric specification in recent literature is the expo-power function introduced by Saha (1993). Using the same notation as Holt and Laury (2002), the expo-power function is defined as

$$
\varphi_{D}(y)=\frac{1}{\alpha}\left(1-\mathrm{e}^{-\alpha y^{1-r}}\right), \text { for } r<1 .
$$

The expo-power functional form converges to a CARA (bounded) function in the limit as $r \rightarrow 0$ and it converges to a power (unbounded) function in the limit as $\alpha \rightarrow 0$. The power function is commonly known as CRRA. ${ }^{8}$ For some $(\alpha, r)$ parameter values the expo-power function is bounded while for other parameter values it is unbounded.

With an expo-power function and $\alpha \neq 0$, a decision theory $\mathrm{D}$ implies that $\left(x^{1-r}+\frac{1}{\alpha} \ln \left(\frac{1}{1-g_{D}(0.5)}\right)\right)^{\frac{1}{1-r}}$ is preferred to $\{\infty, 0.5 ; x\}$, for any given $x$. For example, an expected utility maximizing agent with $\alpha=0.029$ and $r=0.269$ would prefer a certain payoff in amount $\$ 77$ to the lottery $\{\$ \infty, 0.5 ; \$ 0\}$.

The implied risk aversion for the above examples of money transformation functions would be at least as implausible with use of these parametric forms in cumulative prospect theory and rank dependent utility theory as in expected utility theory because in these former two

\footnotetext{
${ }^{8}$ For the case of the expected utility of terminal wealth model, power function utility represents constant relative risk averse preferences, which is the source of the name CRRA.
} 
theories the probability of the high outcome is pessimistically transformed; i.e. $g_{D}(0.5)<0.5$. So, if models of cumulative prospect theory and rank dependent utility theory utilize the same bounded money transformation function as an expected utility model, then if the expected utility model predicts preference of a sure amount $x+L$ to risky lottery $\{G, 0.5 ; x\}$, for all $G$, so do cumulative prospect theory and rank dependent utility theory.

These examples with commonly used parametric utility functions illustrate a general property of all theories in class $\mathbb{I}$ that admit bounded money transformation functions. ${ }^{10}$ The following proposition generalizes the discussion.

Proposition 2. Consider any theory $D$ in class 迥 defined on an unbounded domain that assumes a bounded money transformation function. For any given $x$ there exists a finite $L$ such that $x+L \succ_{D}\{\infty, 0.5 ; x\}$.

Proof: See appendix A.3.

The import of Proposition 2 can be explicated by considering the special case in which the money transformation function $\varphi_{D}$ has an inverse function $\varphi_{D}^{-1}$. In that case the proof of Proposition 2 in appendix A.3 tells us that that if $\varphi_{D}(y) \leq A$ for all $y$ then for any $x>0$ the certain amount of money $z_{D}=\varphi_{D}^{-1}\left(g_{D}(0.5) A+\left(1-g_{D}(0.5)\right) x\right)$ is preferred to a $50 / 50$ lottery that pays $x$ or any positive amount $G$ no matter how large (represented as $\infty$ ). Clearly, $L=z_{D}-x$. Proposition 2 tells us that a bounded money transformation function is a sufficient condition for the implication of implausible risk aversion of type (I*) with decision theories in class $\mathbb{Z}$.

\footnotetext{
${ }^{9}$ Tversky and Kahneman (1992, p. 300) provide the value $g_{D}(0.5)=0.42$ (where, in our notation, $g_{D}$ is the same as their probability weighting function for gains $w^{+}$).

${ }^{10}$ Clearly, Proposition 2 does not apply to expected value theory and the dual theory of expected utility theory because their money transformation functions are (linear and hence) unbounded.
} 


\section{THEORY AND EXPERIMENTS FOR BOUNDED DOMAINS}

\subsection{Does the Original St. Petersburg Paradox Have Empirical Relevance?}

There is a longstanding debate about the relevance of the original version of the St. Petersburg paradox for empirical economics. The claimed bite of the paradox has been based on thought experiments or hypothetical choice experiments in which it was reported that most people say they would be unwilling to pay more than a small amount of money to play a St. Petersburg game with infinite expected value. A traditional dismissal of the relevance of the paradox is based on the observation that no agent could actually offer a real St. Petersburg game for another to play because such an offer would necessarily involve a credible promise to pay unboundedly large amounts of money. Recognition that there is a maximum affordable payment can resolve the paradox for expected value theory. For example, if the maximum affordable payment is (or is believed by the decision maker to be) $\$ 3.3554 \times 10^{7}\left(=\$ 2^{25}\right)$ then the original St. Petersburg lottery is a game that actually pays $\$ 2^{n}$ if $n<25$, and $\$ 2^{25}$ for $n \geq 25$. The expected value of this game is only $\$ 26$, so it would not be paradoxical if individuals stated they would be unwilling to pay large amounts to play the game. If the maximum affordable payment is $\$ 2^{10}=\$ 1,024$ (respectively, $\$ 2^{9}=\$ 512$ ) then the expected value is $\$ 11$ (respectively, $\$ 10$ ). It would be affordable to tests predictions from expected value theory for the last two lotteries with experiments.

\subsection{Does the Generalized St. Petersburg Paradox Have Empirical Relevance?}

It is straightforward to construct affordable St. Petersburg lotteries for any decision theory in class 顷 that assumes unbounded money transformation function. A corollary to Proposition 1 provides a result for an affordable version of the generalized St. Petersburg game for risk preferences that can be represented by functional (6). 
Corollary 1. (An affordable version of the generalized St. Petersburg Game) For any given N, consider a St. Petersburg lottery that pays $x_{n} \in X_{\varphi, h}$ when a fair coin comes up heads for the first time on flip $n$, for $n<N$, and pays $x_{N}$, otherwise. Let $U$ denote the value of functional (6) for this lottery. Then the agent is indifferent between the lottery and receiving a certain amount $\varphi_{D}^{-1}(U)$.

Proof: See appendix A.1.

Let us see what Corollary 1 tells us about one of the commonly used unbounded money transformation functions in the literature, the power function. Suppose that an agent's preferences are assumed to be represented by the expected utility of income model with CRRA or power function utility (or money transformation) function $\varphi_{E U}(x)=x^{1-r} /(1-r)$ for some $r \in(0,1)$. Then the lottery prizes can be set equal to $x_{n}=\left((1-r) 2^{n}\right)^{1 /(1-r)}$ for $n<N+1$, and $x_{N}$ for $n>N$. The corollary implies that the agent with power function coefficient $r$ would be indifferent between getting $((1-r)(N+1))^{1 /(1-r)}$ for sure and playing this game. Figures in the second column of Table 1 are constructed for generalized St. Petersburg games for different values of $r$. Papers on several laboratory and field experiments reported power function (CRRA) estimates in the range 0.44 to $0.67 .{ }^{11}$ The $r=0.5$ value in the table is close to the midpoint of these estimates. As shown in Table 1, an expected utility of income maximizer with power function parameter 0.5 has a certainty equivalent (CE) equal to 9 for the affordable St. Petersburg lottery $\left\{\mathrm{Y}_{4}, 1 / 2_{4}\right\}$ with prizes and $Y_{\infty}=[\ldots, 256,256,64,16,4,1]$, and respective probabilities $1 / 2_{\infty}=\left[\ldots, 2^{-n}, \ldots 2^{-2}, 2^{-1}\right]$.

\footnotetext{
${ }^{11}$ As cited in Holt and Laury (2002, fn. 9, p.1649), CRRA estimates in the range 0.44 to 0.67 were reported by Campo et al. (2000), Chen and Plott (1998), Cox and Oaxaca (1996), Goeree and Holt (2004), and Goeree, et al. (2002, 2003). Harrison, et al. (2007a) reports CRRA estimates within the same range using field experiment data.
} 
For cumulative prospect theory with a value function $x^{\alpha}$ and weighting function $w^{+}(p)=p^{\gamma} /\left(p^{\gamma}+(1-p)^{\gamma}\right)^{1 / \gamma}$ and with reference point 0 (as in Tversky and Kahneman 1992), consider the St. Petersburg game that pays

$$
x_{n}=\left[\frac{\left(2^{1-n}\right)^{\gamma}}{\left(\left(2^{1-n}\right)^{\gamma}+\left(1-\left(2^{1-n}\right)\right)^{\gamma}\right)^{1 / \gamma}}-\frac{\left(2^{-n}\right)^{\gamma}}{\left(\left(2^{-n}\right)^{\gamma}+\left(1-\left(2^{-n}\right)\right)^{\gamma}\right)^{1 / \gamma}}\right]^{1 / \alpha}
$$

if head appears for the first time on the $n$-th flip for $n<N$, and pays $x_{N}$ if the first head appears on any toss $n \geq N+1$. According to cumulative prospect theory, the utility of this game is given by $U_{C P}\left(v_{C P}(n), 2^{-n}\right)=N+1$. Hence, the agent will be indifferent between $\$(\mathrm{~N}+1)^{1 / \alpha}$ for sure and playing this game. Similar results hold for rank dependent utility theory. The last column of Table 1 shows a sequence of payments in an affordable St. Petersburg lottery for cumulative prospect theory models with $\alpha$ and $\gamma$ parameter values reported by Camerer and Ho (1994), Tversky and Kahneman (1992), and Wu and Gonzales (1996). The Wu and Gonzales parameter values of $(\alpha, \gamma)=(0.5,0.71)$ imply that a cumulative prospect theory decision maker with zero reference point has a certainty equivalent of 36 for an affordable St. Petersburg lottery $\left(\left\{\mathrm{Y}_{4}, \mathrm{P}_{4}\right\}\right)$ with prizes $Y_{\infty}=[\ldots, 503,503,220,96,36,4]$. As shown in Table 1, these same parameter values of $(0.5,0.71)$ used for rank dependent utility theory imply that an agent's certainty equivalent for the lottery $\left\{\mathrm{Y}_{4}, 1 / 2_{4}\right\}$ with prizes $Y_{\infty}=[\ldots, 316,316,147,71,32,5]$ is 46.88 .

Finally, for the dual theory of expected utility we report payments involved in a generalized St. Petersburg game for two specifications of the function $f:$ (a) $f(p)=p /(2-p)$ and (b) $f(p)=p^{2}$. The first specification is offered by Yaari as an example that solves the common ratio effect paradox (Yaari 1987, p.105). The second specification is used to demonstrate a rationale for using the Gini coefficient to rank income distributions (Yaari 1987, p.106). Generalized versions of the St. Petersburg game involve payments $2^{n+1}-1$ and $4^{n}$. The affordable versions of the generalized St. Petersburg game are reported in the DU column in 
Table 1. In case (b) with $f(p)=p^{2}$, an example is provided by the sequence of payments $v_{D U}=[\ldots, 342,342,86,22,6,2]$ with expected value of 32 and dual expected utility $U_{D U}\left(v_{D U}, 1 / 2_{\infty}\right)=6$.

\section{$\underline{4.3 \text { A Real Experiment with a Finite St. Petersburg Game }}$}

An experimental design with clear relevance to evaluating the empirical applicability of expected value theory is to offer subjects a finite St. Petersburg bet with highest possible payoff an amount that is known to be affordable for payment by the experimenter. One such experiment, reported by Cox et al. (2007), involved offering subjects the opportunity to decide whether to pay their own money to play nine truncated St. Petersburg bets. One of each subject's decisions was randomly selected for real money payoff. Bets were offered for $N=1,2, \ldots, 9$. Bet $N$ had a maximum of $N$ coin tosses and paid $2^{n}$ euros if the first head occurred on toss number $n$, for $n=1,2, \ldots N$, and paid nothing if no head occurred. The price offered to a subject for playing bet $N$ was 25 euro cents lower than $N$ euros where, of course, $N$ euros was the expected value of bet $N$. An expected value maximizer would accept all of these bets. The experimenter could credibly offer the game to the subjects because the highest possible payoff was $512\left(=2^{9}\right)$ euros for each subject. Cox et al. (2007) report that 47 percent of their subjects' choices were to reject the opportunity to play the St. Petersburg bets. They use a linear mixture model (Harless and Camerer 1994) to estimate whether a risk neutral preference model can characterize the data. Let the letter $a$ denote a subject's response that she accepts the offer to play a specific St. Petersburg game in the experiment. Let $r$ denote rejection of the offer to play the game. The linear mixture model is used to address the specific question whether, for the nine St. Petersburg games offered to their subjects, the risk neutral response pattern $(a, a, a, a, a, a, a, a, a)$ or the risk averse response pattern $(r, r, r, r, r, r, r, r, r)$ is more consistent with the data. Let the stochastic 
preferences with error rate $\varepsilon$ be specified in the following way: (a) if option $\mathrm{Z}$ is preferred then $\operatorname{Prob}($ choose $Z)=1-\varepsilon$; and (b) if option $Z$ is not preferred then Prob(choose Z) $=\varepsilon$. The maximum likelihood point estimate of the proportion of subjects for which risk neutral preferences are rejected in favor of risk averse preferences is 0.49 , with a Wald 90 percent confidence interval of $(0.30,0.67)$. They conclude that 30 percent to 67 percent of the subjects are not risk neutral in this experiment.

\subsection{Plausibility Checks on Empirical Findings with St. Petersburg Games}

Experiments with St. Petersburg games can be designed by following the logic of the discussion in section 4.2. Of course, as that discussion makes clear, one needs a postulated money transformation function and/or postulated probability transformation function to construct the payoffs for the experiment. But that, in itself, does not rule out the possible empirical relevance of the generalized St. Petersburg game, as can be understood from the following. If a researcher concludes, say, that expected utility theory with power function utility (or money transformation) function $\varphi_{E U}(x)=\sqrt{x}$ can rationalize risk preferences on a finite domain of payoffs $[z, Z]$, this opens the question of whether the conclusion is plausible because it implies that the expected utility maximizing agents would accept all finite St. Petersburg bets with prizes $x_{n}=4^{n}, n=1$, $2, \ldots, N$, so long as $4^{N} \leq Z$. The theory implies that the agent with power coefficient $1 / 2$ would reject any sure amount of money up to $\$(N+1)^{2}$ in favor of playing the finite St. Petersburg lottery with a maximum payoff of $N$ coin tosses that pays $\$ 4^{n}$ if the first head occurs on toss number $n$, for $n<N+1$, and pays $\$ 4^{N}$ otherwise. This experiment would be feasible to run for values on $N$ such that $\$ 4^{N}$ is affordable. It would provide an empirical check on plausibility of the conclusion that expected utility theory with square root power function preferences can rationalize the subjects' risky decisions on domain $[z, Z]$. For example, a finite version with $N=$ 
5 of this game that can be credibly tested in the laboratory is reported in Table 1. Let $Y_{5}=[256,64,16,4,1]$ and $1 / 2_{5}=\left[2^{-4}, 2^{-4}, 2^{-3}, 2^{-2}, 2^{-1}\right]$ denote the finite St. Petersburg game that pays $\$ 1$ if a coin lands "head" on the first flip, $\$ 4$ if the coin lands "head" for the first time on the second flip, \$16 if the coin lands "head" for the first time on the third flip, \$64 if the coin lands "head" for the first time on the fourth flip, and $\$ 256$ otherwise (with probability $\left.1-\sum_{n=1}^{4}\left(\frac{1}{2}\right)^{n}\right)$. The expected value of this game is $\$ 23.5$ whereas $U_{E U I}\left(Y_{5}, 1 / 2_{5}\right)=3$. Hence, the expected utility of income model predicts that the agent will prefer getting $\$ 10$ for sure to playing this game. The expected value model, however, predicts that the agent prefers this game to getting \$23 for sure.

For cumulative prospect theory, the last column of Table 1 shows a sequence of payments in a generalized St. Petersburg game. Only payments that are smaller than $\$ 500$ are reported since that is reasonably affordable in an experiment. Suppose for instance that someone has preferences that can be represented by cumulative prospect theory with reference point 0 , $\gamma=0.71$, and $\alpha=0.5$ as reported by $\mathrm{Wu}$ and Gonzales (1996). A finite version of the generalized version of the St. Petersburg game for this case that can be credibly tested in the laboratory is $v_{P T}=[503,220,96,36,4]$. That is, the game pays $\$ 4$ if a coin lands "head" on the first flip, $\$ 36$ if the coin lands "head" for the first time on the second flip, $\$ 96$ if the coin lands "head" for the first time on the third flip, \$220 if the coin lands "head" for the first time on the fourth flip, and $\$ 503$ otherwise. The expected value of this game is $\$ 68.19$ whereas $U_{C P}\left(v_{C P}, 1 / 2_{5}\right)=5.1$. Hence, cumulative prospect theory with the above parameter specifications predicts that the agent will prefer getting \$26 for sure to playing this game. The expected value model, however, predicts that the agent prefers the game to getting $\$ 68$ for sure. Table 1 also reports examples of lotteries and predictions by rank dependent utility theory and dual theory of expected utility, as discussed in section 4.2. 


\section{$\underline{4.5 \text { Plausibility Checks on Empirical Findings with Binary Lotteries }}$}

Proposition 2 can provide a researcher with checks on the empirical plausibility of estimates of risk aversion parameters on a finite domain $[z, Z]$. Using the notation of the proposition, questions that are clearly relevant to a finite domain involve payoff amounts $x$ and $x+L$ and $G$, all in the domain of interest, that imply $x+L$ for sure is preferred to $\{G, 0.5 ; x\}$. Implications such as these provide plausibility checks on reported parameter estimates.

Table 2 presents some implications of two money transformation (or utility) functions using parameter estimates for three experiments with small stakes lotteries reported in the literature. The parameter estimates are taken from Harrison and Rutström (2008, Table 8, p. 120). Unlike the discussion in section 3.2 above, we here examine the implications of estimated parametric money transformation functions only on the local domains of the data samples used in estimation of the parameters. As shown at the top of Table 2, data are from experiments reported by Holt and Laury (2005), Hey and Orme (1994), and Harrison and Rutström (2008). As shown just below the top of the table, parameter estimates from two functional forms are used: CRRA and expo-power (EP). As shown at the next level in the table, estimates based on two theories are used: expected utility of income models (EU) and rank dependent utility models (RD).

The entries in the first and third columns of Table 2 convey the following information. The third column reports parameter estimates for a rank dependent utility model with power functions for both the money transformation and probability transformation functions. Data from the experiment reported in Holt and Laury (2005) yield the parameter estimate $\hat{\rho}=0.85$ for the money transformation function and the parameter estimate $\hat{\gamma}=1.46$ for the probability transformation function. With these parameters, the rank dependent utility model implies that $\$ 0.40$ for sure (in column 3 ) is preferred to the lottery $\{\$ 77,0.5 ; \$ 0\}$ (in column 1 ). It seems to us likely that almost all people would have risk preferences that are inconsistent with this prediction 
and, in that sense, that the estimated parametric utility function is implausible. Importantly, the prediction that $\$ 0.40$ for sure is preferred to $\{\$ 77,0.5 ; \$ 0\}$ is clearly testable and, therefore, a conclusion about plausibility or implausibility of the estimated model can be based on data not mere opinion.

Estimation of the CRRA parameter using the expected utility of income model and data from Holt and Laury (2005) yields $\hat{r}=0.76$. With this parameter, as reported in the second column of Table 2, $\$ 4.30$ for sure is preferred to the lottery $\{\$ 77,0.5 ; \$ 0\}$. The fourth and fifth columns of Table 2 report parameter estimates for the expo-power (EP) money transformation function. The parameter estimates imply that $\$ 8.60$ for sure is preferred to the lottery $\{\$ 77,0.5 ; \$ 0\}$ for the rank dependent utility model. The preferred sure amount of money is $\$ 15.80$ in case of the expected utility of income model.

Table 2 uses point estimates of parameters from three data sets and four combinations of money transformation and probability transformation functions to derive implied preferences for sure amounts of money (in all columns except the first) over binary lotteries (in the first column). All of these implied preferences are stated on domains that are the same or smaller than those for the data samples. Furthermore, all of these implied preferences are testable with real, affordable experiments. Conducting such tests would provide data to inform researchers' decisions about whether the estimated parametric forms provide plausible or implausible characterizations of the risk attitudes of the subjects in experiments. Finally, similar experiments can be designed with binary lotteries based on any parameter estimates within the $90 \%$ confidence limits of the estimation if a researcher wants to thoroughly explore the plausibility question.

In preceding sections, we have explored testable implications for empirical plausibility of parametric forms of decision theories in class $\mathbb{T}$. Some recent studies have identified patterns of risk aversion, known as calibration patterns, that can be used to test plausibility of theories under risk without any parametric specifications. Concavity calibrations involve certain types of 
patterns of choices that target decision theories under risk that assume concave money transformation (or utility) functions (Rabin 2001, Neilson 2004, Cox and Sadiraj 2006, Rubinstein 2006). Convexity calibrations, on the other hand, involve patterns of risk aversion that apply to theories that represent risk aversion with probability transformation functions (Cox, et al. 2007). The following three sections summarize what is currently known about the empirical validity of patterns of risk aversion underlying calibration propositions.

\subsection{Do Concavity Calibrations of Payoff Transformation (or Utility) Functions Have Empirical}

\section{$\underline{\text { Relevance? }}$}

Cox et al. (2007) report an experiment run in Calcutta, India to test the empirical validity of a postulated pattern of small stakes risk aversion that has implications for cumulative prospect theory, rank dependent utility theory, and all three expected utility models discussed in Cox and Sadiraj (2006), the expected utility of terminal wealth model, the expected utility of income model, and the expected utility of initial wealth and income model.

Subjects in the Calcutta experiment were asked to choose between a certain amount of money, $x$ rupees (option $\mathrm{B}$ ) and a binary lottery that paid $x-20$ rupees or $x+30$ rupees with equal probability (option A) for values of $x$ from a finite set $\Omega$. Subjects were informed that one of their decisions would be randomly selected for payoff. The amount at risk in the lotteries (50 rupees) was about a full day's pay for the subjects in the experiment. By Proposition 1 in Cox et al. (2007), if a subject chooses option B for at least four sequential values of $x$ then calibration of the revealed pattern of small stakes risk aversion implies behaviorally implausible large stakes risk aversion. They call any choice pattern that meets this criterion a "concavity calibration pattern" and test a null hypothesis that the data are not characterized by concavity calibration patterns against an alternative that includes them. To conduct the test, Cox et al. (2007) applied a linear mixture model similar to that described in section 4.3. The reported point estimate for the proportion of the subjects in the Calcutta experiment that made choices for which 
expected utility theory, rank dependent utility theory, cumulative prospect theory (with 0 reference point payoff) imply implausible large stakes risk aversion was 0.495 , with Wald 90 percent confidence interval of $(0.289,0.702)$. They conclude that 29 percent to 70 percent of the subjects made choices that, according to three theories of risky decision making, can be calibrated to imply implausible large stakes risk aversion. According to Proposition 2 in Cox et al. (2007), this conclusion applies to all theories in class $\mathbb{\text { I }}$ that represent risk preferences with concave transformations of payoffs. Thus the conclusion applies to all expected utility models regardless of whether they specify full asset integration (the terminal wealth model), no asset integration (the income model), or partial asset integration (variants of the initial wealth and income model).

Prospect theory can be immunized to concavity calibration critique by introducing variable reference points set equal to the $x$ values in the Calcutta experiments (Wakker 2005). The variable reference points do not, however, immunize prospect theory to other tests with data from the experiment because they imply that a subject will make the same choice (of the lottery or the certain payoff), for all values the sure payoff $x$. Cox et al. report that the likelihood ratio test rejects this "non-switching hypothesis" in favor of an alternative that allows for one switch at 5 percent significance level. Adding possible choice patterns with more than one switch to the alternative hypothesis would also lead to rejection of the non-switching hypothesis. Hence, variable reference points do not rescue cumulative prospect theory from inconsistency with the data from the experiment.

\section{$\underline{4.7}$ Do Convexity Calibrations of Probability Transformation Functions Have Empirical}

\section{$\underline{\text { Relevance? }}$}

Cox et al. (2007) demonstrate that the problem of possibly implausible implications from theories of decision making under risk is more generic than implausible (implications of) decreasing marginal utility of money by extending the calibration literature in their Proposition 2 to include the implications of convex transformations of decumulative probabilities used to model risk 
aversion in the dual theory. They report another experiment run in Magdeburg, Germany in which subjects were asked to make nine choices between pairs of lotteries. Subjects were informed that one of their decisions would be randomly selected for payoff. Decision task $i$, for $i=1,2, \ldots, 9$, presented a choice between lottery A that paid $€ 40$ with probability $i / 10$ and $€ 0$ with probability $1-i / 10$ and lottery $B$ that paid $€ 40$ with probability $(i-1) / 10, € 10$ with probability $2 / 10$, and $€ 0$ with probability $1-[(i-1+2) / 10]$. By Proposition 2 in Cox et al. (2007), if a subject chooses lottery B for at least seven sequential values of the probability index $i$ then calibration of the revealed pattern of small stakes risk aversion implies implausible large stakes risk aversion for the dual theory. They call any choice pattern that meets this criterion a "convexity calibration pattern" and test the null hypothesis that the data are not characterized by convexity calibration patterns against an alternative that includes them. Again applying a linear mixture model, Cox et al. (2007) report that the linear mixture model yields a point estimate of 0.81 and Wald 90 percent confidence interval of $(0.66,0.95)$ for the proportion of subjects for which the dual theory implies implausible risk aversion. Thus the data are consistent with the conclusion that 66 percent to 95 percent of the subjects made choices that, according to the dual theory, can be calibrated to imply implausible large stakes risk aversion.

\subsection{Is the Expected Utility of Terminal Wealth Model More (or Less) Vulnerable to Calibration}

\section{Critique than Other Theories?}

Rabin (2000) initiated recent literature on the large stakes risk aversion implications implied by calibration of postulated patterns of small stakes risk aversion. His analysis is based on the supposition that an agent will reject a small stakes gamble with equal probabilities of $50 \%$ of winning or losing relatively small amounts, and that the agent will do this at all initial wealth levels in some large interval. For example, Rabin demonstrated that if an agent would reject a $50 / 50$ bet in which she would lose $\$ 100$ or gain $\$ 110$ at all initial wealth levels up to $\$ 300,000$ 
then the expected utility of terminal wealth model implies that, at an initial wealth level of $\$ 290,000$, that agent would also reject a $50 / 50$ bet in which she would lose $\$ 6,000$ or gain $\$ 180$ million.

Rabin (2000) and Rabin and Thaler (2001) stated strong conclusions about implausible risk aversion implications for expected utility theory implied by their supposed patterns of small stakes risk aversion but reported no experiments supporting the empirical validity of the suppositions. Their conclusions about expected utility theory were taken quite seriously by some scholars (Kahneman 2003, Camerer and Thaler 2003) and by a Nobel Prize committee (Royal Swedish Academy of Sciences 2002, p. 16), despite the complete absence of data consistent with the supposed patterns of small stakes risk aversion underlying the concavity calibrations. It is ironic that, in this heyday of behavioral economics, strong conclusions about the behavioral plausibility of theory could be drawn without any actual observations of behavior.

As explained by Cox and Sadiraj (2006), observations of behavior consistent with the pattern of risk aversion supposed in Rabin's concavity calibration would have limited implications for risky decision theory because they would have no implications for expected utility models other than the terminal wealth model nor for other theories in class $\mathbb{T}$ in which income rather than terminal wealth is postulated as the argument of functional (6). Furthermore, an experiment that could provide empirical support for Rabin's supposition would have to be conducted with a within-subjects design, as we shall explain after first explaining problems with across-subjects experiments in the literature.

Barberis et al. (2003) report an across-subjects, hypothetical experiment with a 50/50 lose $\$ 500$ or gain $\$ 550$ bet using as subjects MBA students, financial advisers, investment officers, and investor clients. They report that about half of the subjects stated they would be unwilling to accept the bet. They do not report wealth data for these subjects nor the relationship, if any, between subjects' decisions and their wealth levels; therefore the relation between the subjects' decisions and the supposed pattern of risk aversion used in concavity calibration propositions is 
unknown. Barberis et al. (2003) also report an across-subjects, real experiment with a 50/50 lose $\$ 100$ or gain $\$ 110$ bet using MBA students as subjects. They report that only 10 percent of the subjects were willing to play the bet. No wealth data are reported for these subjects either.

It is straightforward to show that any across-subjects experiment involving one choice per subject cannot provide data that would support the conclusion of implausible risk aversion. Suppose one has a sample from an experiment (like the two Barberis et al. 2003 experiments) in which each of $N$ subjects is asked to make one decision about accepting or rejecting a 50/50 lose $\$ 100$ or gain $\$ 110$ bet. Suppose that the initial wealth level of every subject is observed and that these wealth levels vary across a large range, say from a low of $\$ 100$ to a high of $\$ 300,000$. Would such a data sample provide support for any conclusion about the expected utility of terminal wealth model? Without making other assumptions about preferences, the answer is clearly "no" as we next explain.

Suppose that we observe individual wealth levels $\tilde{w}_{j} \in[100,300 K], j=1,2, \ldots, N$, for each of $N$ individuals and that every one of them rejects the 50/50 lose $\$ 100$, gain $\$ 110$ bet. Can they all be expected utility of terminal wealth maximizers with globally plausible risk aversion? Yes, and the following equation can be used to generate $N$ utility functions with parameters $a_{j}$ and $r_{j}$, each of which implies indifference between accepting and rejecting the bet at the observed individual wealth levels:

$$
2=\left(1-\frac{100}{\tilde{w}_{j}-a_{j}}\right)^{r_{j}}+\left(1+\frac{110}{\tilde{w}_{j}-a_{j}}\right)^{r_{j}}, a_{j} \leq \tilde{w}_{j}-100
$$

Any ordered pair of parameters $\left(a\left(\tilde{w}_{j}\right), r\left(\tilde{w}_{j}\right)\right)$ below the graph of the level set of this equation can be used to construct a utility function

$$
u_{j}\left(\tilde{w}_{j}+y\right)=\left(-a\left(\tilde{w}_{j}\right)+\tilde{w}_{j}+y\right)^{r\left(\tilde{w}_{j}\right)}
$$


that implies rejection of the bet for an expected utility of terminal wealth maximizer with initial wealth $\tilde{w}_{j}$ and money transformation function given by (17). But each of these utility functions exhibits plausible risk aversion globally. Therefore, the empirical relevance of Rabin's concavity calibration for the expected utility of terminal wealth model cannot be tested with an acrosssubjects experiment.

The empirical validity of Rabin's concavity calibration for the expected utility of terminal wealth model could, however, be tested with a within-subjects experiment. Let subject $j$ have initial wealth $w_{j}$ at the beginning of the experiment. In round $t$ of the experiment, give subject $j$ an amount of money $x_{t}$ and an opportunity to play a 50/50 bet with loss of 100 or gain of 110 . Choose the set $X$ of values for $x_{t}$ so that there are enough observations covering a sufficiently large range that concavity calibration can bite. An example of suitable specifications of the set $X$ are provided by the sets of certain income payoffs used in the Calcutta experiment reported in Cox et al. (2007) and summarized above. Consider the set of certain payoff $x$ values used in the Calcutta experiment; define $X=\{100,1 K, 2 K, 4 K, 5 K, 6 K\}$ and let $x_{t}$ denote the value in position $t$ in this set. Using subject $j$ 's (observed) initial wealth $w_{t}$ at the beginning of the experiment, and the controlled values $x_{t}, t=1,2, \ldots, 6$, define subject $j$ 's variable initial wealth level during the experiment as $\omega_{j t}=w_{j}+x_{t}$. At round $t$ in the experiment, give the subject $X_{t}$ and then ask her whether she wants to accept the 50/50 gamble with loss amount 100 and gain amount 110 . If the answer is "no" for at least four sequential values of $x$ then Proposition 1 in Cox et al. (2007) or Rabin's (2000) concavity calibration proposition imply implausible risk aversion for the expected utility of terminal wealth model. Therefore this type of 
"pay- $x$-in-advance," within-subjects experiment could support, or fail to support, the empirical relevance of Rabin's concavity calibration supposition for the terminal wealth model. ${ }^{12}$

\section{SUMMARY IMPLICATIONS FOR THEORIES OF RISKY DECISIONS}

Some implications for theories of decision making under risk are straightforward while others are nuanced.

\subsection{Decision Theories on Unbounded Domain Have Implausible Implications}

One implication is that all theories in class $刃$ 回 have the same problems with respect to the plausibility of modeling decisions under risk on an unbounded domain. This conclusion follows from the demonstration that, on an unbounded domain, theories in class 7 are characterized by either the generalized St. Petersburg paradox or implausible aversion to risk of type (I*). This raises doubts about the plausibility of classic developments of expected utility theory for risky decisions (Pratt 1964, Arrow 1971). But this plausibility critique of the theory is not confined to expected utility theory; instead, it applies to all theories in a class that contains cumulative prospect theory, rank dependent utility theory, and dual theory of expected utility (as well as expected utility theory). In this sense, the fundamental problems shared by these theories may be more significant than their much-touted differences.

\subsection{Implications for Theory and its Applications on Bounded Domains}

Theories of risky decisions defined on bounded domains can be characterized by the generalized St. Petersburg paradox or by implausible large stakes risk aversion or by neither problem.

12 In contrast, this type of experiment could not produce data that would have a calibration-pattern implication for any of the other models discussed above for which income, not terminal wealth is the argument of the utility functional (Cox and Sadiraj 2006). However, this type of experiment would have a testable implication for all other models in class $\mathbb{T}$ : (a) always choose the risky option with EV theory; or (b) always choose the same option with other theories. 
Conclusions for theory on bounded domains are more nuanced, and more complicated, but they are empirically testable.

Concavity calibration of postulated patterns of risk aversion have implausible large stakes risk aversion implications for all theories in class 㯊 that incorporate decreasing marginal utility of money except for specific versions of prospect theory that postulate variable reference points (which are rejected by testing the data). Implausible implications for theory following from calibrating postulated patterns of risk aversion are not confined to theories with decreasing marginal utility of money. The dual theory of expected utility, characterized by constant marginal utility of money, can be critiqued with convexity calibration of the probability transformation that exclusively incorporates risk aversion into this theory.

Whether or not critiques with the generalized St. Petersburg paradox or (calibrated) implausible large stakes risk aversion have bite for theories defined on bounded domains are empirical questions. The reason for this is apparent: people may accept feasible St. Petersburg bets and/or they may not reject the small stakes bets postulated in calibrations. If both those outcomes were observed, the St. Petersburg paradox and calibration critiques would have no implication of implausible theory for bounded domains. To date, the empirical evidence is limited.

As discussed above, even on very large bounded domains, expected values for St. Petersburg bets are quite small, on the order of $\$ 25$, which (for what it's worth) is consistent with commonly reported subjects' statements about willingness to pay to play the bets in hypothetical experiments. In one real payoff experiment with finite St. Petersburg bets reported by Cox et al. (2007), 30 percent to 67 percent of the subjects revealed risk preferences that were inconsistent with the expected value model.

There is not yet any existing study that supports the conclusion that terminal wealth models are more vulnerable to calibration critique than income models. There are various misstatements in the literature about the existence of data supporting Rabin's (2000) supposition 
that an agent will reject a given small stakes bet at all initial wealth levels in a wide interval. In fact, there is no test of Rabin's supposition in the literature. Furthermore, a test of this supposition would, in any case, have no implications for models in which income rather than terminal wealth is the argument of utility functionals (Cox and Sadiraj 2006).

The within-subjects Calcutta experiment with concavity calibration reported by Cox et al. (2007) has implications for all three expected utility models, rank dependent utility theory, and the original version of cumulative prospect theory with constant reference point equal to zero income (Tversky and Kahneman 1992). This was a within-subjects, real payoff experiment. In the Calcutta experiment, 25 percent to 62 percent of the subjects made patterns of small stakes risky choices for which expected utility theory, rank dependent utility theory, and prospect theory (with zero reference point payoff) imply implausible large stakes risk aversion. Variable reference points can be incorporated into prospect theory in ways that immunize the theory to concavity calibration critique with this experimental design. But the testable implication of this version of prospect theory has a high rate of inconsistency with data from the Calcutta experiment and is rejected in favor of the "calibration pattern" by a likelihood ratio test.

The Madgeburg experiment with convexity calibration for probability transformations (Cox et al. 2007) has implications for the dual theory of expected utility that has constant marginal utility of money and incorporates risk aversion solely through non-linear transformation of probabilities. In this experiment, 66 percent to 95 percent of the subjects made patterns of risky choices for which the dual theory of expected utility implies implausible large stakes risk aversion.

We conclude that, together, the Calcutta concavity calibration experiment and Magdeburg convexity calibration experiment provide data that suggest skepticism about the plausibility of popular theories of decision making for risky environments. However, more experiments and larger samples are needed to arrive at definitive conclusions about the empirical relevance of the calibration propositions. One thing that is clear is that the traditional focus on decreasing 
marginal utility of money as the source of implausible implications from calibration of postulated patterns of risk aversion is wrong; modeling risk aversion with probability transformations also can produce implausible implications from calibration.

Empirical research leading to conclusions that estimated parametric forms of utility functionals can represent subjects' behavior in risky decision making can be checked for plausibility by applying research methods explained here. Two types of questions can be posed. First, does the estimated parametric form survive testing with St. Petersburg lotteries that can be derived from the parametric form using methods explained above? Second, does the estimated parametric form of a utility functional survive experimental testing with binary lottery designs that can be derived from the parametric form using methods explained above? If the answer to either question is "no" then the conclusion that the estimated utility functional can rationalize risk taking behavior is called into question. 


\section{ACKNOWLEDGMENT}

We thank Glenn W. Harrison and Nathanial T. Wilcox for helpful comments and suggestions.

Financial support was provided by the National Science Foundation (grant numbers DUE0622534 and IIS-0630805).

\section{REFERENCES}

Arrow, K. J. (1971). Essays in the Theory of Risk-Bearing. Markham, Chicago.

Barberis, N., Huang, M. and Thaler, R. (2003). Individual Preferences, Monetary Gambles and the Equity Premium. NBER Working Paper 9997.

Bernoulli, D. (1738). Specimen Theoriae Novae de Mensura Sortis, Commentarii Academiae Scientiarum Imperialis Petropolitanae 5, 175-192. English translation (1954): Exposition of a New Theory on the Measurement of Risk," Econometrica 22, 23-36.

Camerer, C. F. and Ho, T. (1994). Violations of the Betweenness Axiom and Nonlinearity in Probability. Journal of Risk and Uncertainty 8, 167-196.

Campo, S., Perrigne, I. and Vuong, Q. (2000). Semi-Parametric Estimation of First-Price Auctions with Risk Aversion. Working paper, University of Southern California.

Chen, K. and Plott, C. R. (1998). Nonlinear Behavior in Sealed Bid First-Price Auctions. Games and Economic Behavior 25, 34-78.

Cox, J. C. and Oaxaca, R. L. (1996). Is Bidding Behavior Consistent with Bidding Theory for Private Value Auctions? In R. M. Isaac, (Ed.), Research in Experimental Economics V6. Greenwich, CT: JAI Press.

Cox, J. C. and Sadiraj, V. (2006). Small- and Large-Stakes Risk Aversion: Implications of Concavity Calibration for Decision Theory. Games and Economic Behavior $56,45-60$. 
Cox, J. C., Sadiraj, V., Vogt, B. and Dasgupta, U. (2007). Is There A Plausible Theory for Risky Decisions? Experimental Economics Center Working Paper 2007 - 05, Georgia State University.

Cox, J. C., Smith, V.L. and Walker, J. M. (1982). Auction Market Theory of Heterogeneous Bidders. Economics Letter 9, 319-325.

Goeree, J. K. and Holt, C. A. (2004). A Model of Noisy Introspection. Games and Economic Behavior 47, 365 - 382.

Goeree, J. K., Holt, C. A. and Palfrey, T. (2002). Quantal Response Equilibrium and Overbidding in Private-Value Auctions. Journal of Economic Theory 104, 247272.

Goeree, J. K., Holt, C. A. and Palfrey, T. (2003). Risk Averse Behavior in Generalized Matching Pennies Games. Games and Economic Behavior 45, 97-113.

Harless, D. and Camerer, C. F. (1994). The Predictive Utility of Generalized Expected Utility Theories. Econometrica 62, 1251-90.

Harris, M. and Raviv, A. (1981). Allocation Mechanisms and the Design of Auctions. Econometrica, 49, 1477-1499.

Harrison, G. W., Lau M. and Rutström (2007a). Estimating Risk Attitudes in Denmark: a Field Experiment. Scandinavian Journal of Economics 109, 341 -368.

Harrison, G. W., List, J. and Towe, C. (2007b). Naturally Occurring Preferences and Exogenous Laboratory Experiments: A Case Study of Risk Aversion. Econometrica 75, 433-458. Harrison, G. W. and Rutström, E. E. (2008). Risk Aversion in the Laboratory. In J.C. Cox and G.W. Harrison (eds.), Risk Aversion in Experiments (Greenwich, CT: JAI Press, Research in Experimental Economics, Volume 12).

Heinemann, F. (2008). Measuring Risk Aversion and the Wealth Effect. In J.C. Cox and G.W. Harrison (eds.), Risk Aversion in Experiments (Greenwich, CT: JAI Press, Research in Experimental Economics, Volume 12). 
Hey, J. and Orme, C. (1994). Investigating Generalizations of Expected Utility Theory Using Experimental Data. Econometrica 62, 1291 - 1326.

Holt, C. A. Jr. (1980). Competitive Bidding for Contracts Under Alternative Auction Procedures. Journal of Political Economy 88, 433-445.

Holt, C. A. and Laury, S. (2002). Risk Aversion and Incentive Effects. American Economic Review 92, 1644- 1655.

Holt, C. A. and Laury, S. (2005). Risk Aversion and Incentive Effects: New Data without Order Effects. American Economic Review 95, 902 - 912.

Kahneman, D. (2003). A Psychological Perspective on Economics. American Economic Review Papers and Proceedings 93, 162-168.

Kahneman, D. and Tversky, A. (1979). Prospect Theory: An Analysis of Decision under Risk. Econometrica 47, 263-291.

Laffont, J. J. (1989). The Economics of Uncertainty and Information. MIT Press, Cambridge, MA: MIT Press.

Maskin, E. and Riley, R. (1984). Optimal Auctions with Risk Averse Buyers. Econometrica 52, 1473-1518.

Milgrom, P. R. and Weber, R. J. (1982). A Theory of Auctions and Competitive Bidding. Econometrica 50, 1089-1122.

Moore, J. (1984). Global Incentive Constraints in Auction Design. Econometrica 52, 1523-1536.

Neilson, W. S. (2001). Calibration Results for Rank-Dependent Expected Utility. Economics Bulletin 4, 1-5.

von Neumann, J. and Morgenstern, O. (1947). Theory of Games and Economic Behavior. Princeton NJ: Princeton University Press.

Pratt, J. W. (1964). Risk Aversion in the Small and in the Large. Econometrica 32, $122-$ 136. 
Quiggin, J. (1982). A Theory of Anticipated Utility. Journal of Economic Behavior and Organization 3, 323-343.

Quiggin, J. (1993). Generalized Expected Utility Theory. The Rank-Dependent Model. Boston: Kluwer Academic Publishers.

Rabin, M. (2000). Risk Aversion and Expected Utility Theory: A Calibration Theorem. Econometrica 68, 1281-1292.

Rabin, M. and Thaler, R. H. (2001). Anomalies: Risk Aversion. Journal of Economic Perspectives 15, 219-232.

Rieger, M. O. and Wang, M. (2006). Cumulative Prospect Theory and the St. Petersburg Paradox. Economic Theory 28, $665-679$.

Riley, J. G. and Samuelson, W. F. (1981). Optimal Auctions. American Economic Review 71, 381-392.

Rubinstein, A. (2006). Dilemmas of An Economic Theorist. Econometrica 74, 865-883.

Samuelson, P. A. (1977). St. Petersburg Paradoxes: Defanged, Dissected, and Historically Described. Journal of Economic Literature 15, 24 - 55.

Tversky, A. and Kahneman, D. (1992). Advances in Prospect Theory: Cumulative Representation of Uncertainty. Journal of Risk and Uncertainty 5, 297-323.

Saha, A. (1993). Expo-Power Utility: A 'Flexible' Form for Absolute and Relative Risk Aversion. American Journal of Agricultural Economics 75, 905-913

Wakker, P. P. (2005). Formalizing Reference Dependence and Initial Wealth in Rabin's Calibration Theorem. working paper, Econometric Institute, Erasmus University, Rotterdam.

Wu, G. and Gonzalez, R. (1996). Curvature of the Probability Weighting Function. Management Science 42, 1676-1690.

Yaari, M. E. (1987). The Dual Theory of Choice under Risk. Econometrica 55, 95-115. 


\section{APPENDIX}

A.1. Lemma Let functions $h:[0,1] \rightarrow[0,1], \quad$ s.t. $h\left(p_{n}, P_{\infty}^{-n}\right) \neq 0$ for all $p_{n} \neq 0$, and $\varphi: \mathfrak{R} \rightarrow \mathfrak{R}$, be given. If $\varphi$ is unbounded from above then

(a) for all $n \in N$, there exists $x_{n} \in N$ such that $\varphi\left(x_{n}\right) \geq 1 / h\left(1 / 2^{n},\left(1 / 2_{\infty}\right)^{-n}\right)$, and

(b) $\sum_{n \in \mathbb{N}} h\left(1 / 2^{n},\left(1 / 2_{\infty}\right)^{-n}\right) \varphi\left(x_{n}\right)=\infty$

Proof. (a) It follows from $h\left(1 / 2^{n},\left(1 / 2_{\infty}\right)^{-n}\right) \neq 0$, for all $n \in N$, and by definition of a function being unbounded from above.

(b) Part (a) and $h\left(1 / 2^{n},\left(1 / 2_{\infty}\right)^{-n}\right)>0$ implies $\varphi\left(x_{n}\right) \times h\left(1 / 2^{n},\left(1 / 2_{\infty}\right)^{-n}\right) \geq 1$, hence (b) is true.

\section{A.2. Corollary: An Affordable Version of the Generalized St. Petersburg game}

Let an agent's preferences, $\succeq_{\varphi, h}$ on lottery space be represented by functional (6) with an unbounded money transformation function $\varphi$ and probability transformation function $h$. Define $X_{\varphi, h}=\left\{x_{n} \mid n \in N, x_{n} \geq \sup \left\{\varphi^{-1}\left(1 / h\left(1 / 2^{n},\left(1 / 2_{\infty}\right)^{-n}\right)\right)\right\}\right\}$. For any given $N$,

$$
\varphi^{-1}(N+1) \succeq_{\varphi, h}\left\{x_{\infty}, 1 / 2_{\infty}\right\}_{N}
$$

where $\left\{x_{\infty}, 1 / 2_{\infty}\right\}_{N}$ is a St. Petersburg game that pays $x_{n} \in X_{\varphi, h}$ when a fair coin comes up heads for the first time on flip $n$, for $n<N$ and $x_{N}$ otherwise.

Proof. Note that

$\left.U\left(\left\{x_{\infty}, 1 / 2_{\infty}\right\}_{N}\right)=\sum_{n=1 . . N} h\left(1 / 2^{n},\left(1 / 2_{\infty}\right)^{-n}\right) \varphi\left(x_{n}\right)+\varphi\left(x_{N+1}\right) \sum_{n>N} h\left(1 / 2^{n},\left(1 / 2_{\infty}\right)^{-n}\right)\right)=N+1$

\section{A.3. Implausible Risk Aversion of type (I*)}

Let an agent's preferences on lottery space be represented by represented by a theory $D$ with functional (6) with a bounded money transformation function. For any given $x$ there exists a $L$ such that $x+L \succeq_{D}\{\infty, 0.5 ; x\}$.

Proof. Let $\varphi$ be the money transformation function and $\mathrm{g}(0.5)$ be the transformed probability of 0.5 under decision theory D. Function $\varphi$ is bounded from above and positively monotonic, so: there exists and A such that (i) $A=\sup _{x}\{\varphi(x)\}$, and (ii) $\lim _{x \rightarrow \infty} \varphi(x)=A$. For any given $\mathrm{x}$, take 
$\varepsilon=(A-\varphi(x))(1-g(0.5))>0$ and apply (ii) to find a $z_{x}$ s.t. $\varphi\left(z_{x}\right)>A-\varepsilon$. To complete the proof take $L=z_{x}-x$, substitute the expression of $\varepsilon$ in the last inequality and verify that $\varphi(x+L)>A g(0.5)+\varphi(x)(1-g(0.5))$. 
Table 1. Payments in Finite St. Petersburg Lotteries ${ }^{\mathrm{a}}$

\begin{tabular}{|c|c|c|c|c|c|}
\hline \multicolumn{6}{|c|}{$\mathrm{EV}: x_{n}=2^{n},[2,4,8,16,32,64,128,256,512],(\mathrm{EV}=10)$} \\
\hline \multicolumn{2}{|r|}{ Power Function EU } & \multirow{2}{*}{$\frac{\mathrm{DU}}{x_{n}=1 / h_{D}\left(2^{-n}\right)}$} & \multicolumn{3}{|r|}{$\mathrm{CP}$ and $\mathrm{RD}\left(\varphi(x)=x^{\alpha}\right)$} \\
\hline $\mathrm{R}$ & $x_{n}=\left((1-r) 2^{n}\right)^{1 /(1-r)}$ & & $\alpha$ & $\gamma$ & $x_{n}$ \\
\hline 0.1 & $\begin{array}{c}\mathrm{L}=[2,5,9,20,42,91,196,422] \\
(\mathrm{CE}(\mathrm{L})=10.56 ; \mathrm{EV}(\mathrm{L})=12.19)\end{array}$ & $\begin{array}{c}(\mathrm{f}(\mathrm{p})=\mathrm{p} /(2-\mathrm{p})) \\
\mathrm{L}=[2,6,14,30,62,126,254,510] \\
(\mathrm{CE}(\mathrm{L})=9.6 ; \mathrm{EV}(\mathrm{L})=16)\end{array}$ & $0.88^{\mathrm{d}}$ & $0.62^{\mathrm{d}}$ & $\begin{aligned} \mathrm{L}= & {[2,10,17,24,35,50,75,115,180,284,454] } \\
& (\mathrm{CE}(\mathrm{L})=18.50 ; \mathrm{EV}(\mathrm{L})=11.11)\end{aligned}$ \\
\hline 0.5 & $\begin{array}{c}\mathrm{L}=[1,4,16,64,256] \\
(\mathrm{CE}(\mathrm{L})=9 ; \mathrm{EV}(\mathrm{L})=23.5)\end{array}$ & $\begin{array}{c}\left(\mathrm{f}(\mathrm{p})=\mathrm{p}^{2}\right) \\
\mathrm{L}=[2,6,22,86,342] \\
(\mathrm{CE}(\mathrm{L})=6 ; \mathrm{EV}=32)\end{array}$ & $0.5^{\mathrm{e}}$ & $0.71^{\mathrm{e}}$ & $\begin{array}{c}\mathrm{L}=[4,36,96,220,503] \\
(\mathrm{CE}(\mathrm{L})=46.88 ; \mathrm{EV}(\mathrm{L})=68.19)\end{array}$ \\
\hline 0.67 & $\begin{aligned} \mathrm{L} & =[1,3,19,155] \\
(\mathrm{CE}(\mathrm{L}) & =6.45 ; \mathrm{EV}(\mathrm{L})=23)\end{aligned}$ & & $0.37^{\mathrm{b}}$ & $0.56^{\mathrm{f}}$ & $\begin{array}{c}\mathrm{L}=[4,391] \\
(\mathrm{CE}(\mathrm{L})=61.62 ; \mathrm{EV}(\mathrm{L})=197.5)\end{array}$ \\
\hline
\end{tabular}

${ }^{a}$ A prize vector of length $\mathrm{k}$ means the lottery pays the $n$-th coordinate when head appears for the first time on flip $n$ for $n<k$, and $X_{k}$ otherwise.

${ }^{b}$ The estimate of alpha is the estimate of Wu and Gonzalez (p.1686) using Camerer and Ho (1994) data.

c (field data) Campo et al. (2000).

d Tversky and Kahneman (1992).

e Wu and Gonzalez (1996).

${ }^{\mathrm{f}}$ Camerer and Ho (1994). 
Table 2. Predictions for Binary Lotteries Using Parameter Point Estimates from Small Stakes Data

\begin{tabular}{|c|c|c|c|c|c|c|c|c|c|c|c|c|}
\hline & \multicolumn{4}{|c|}{ Holt and Laury (2005) } & \multicolumn{4}{|c|}{ Hey and Orme (1994) } & \multicolumn{4}{|c|}{$\begin{array}{l}\text { Harrison and Rutström (2008) } \\
\text { replication of } \\
\text { Hey and Orme (1994) }\end{array}$} \\
\hline $\begin{array}{c}\text { Binary } \\
\text { Lotteries }^{a}\end{array}$ & $\begin{array}{c}\text { EU } \\
\hat{r}=0.76\end{array}$ & $\begin{array}{l}\mathrm{RD} \\
\hat{\rho}=0.85 \\
\hat{\gamma}=1.46^{\mathrm{b}}\end{array}$ & $\begin{array}{c}\text { EU } \\
\hat{r}=0.4 \\
\hat{\alpha}=0.07\end{array}$ & $\begin{array}{c}\mathrm{RD} \\
\hat{\rho}=0.26 \\
\hat{\alpha}=0.02^{\mathrm{b}} \\
\hat{\gamma}=0.37\end{array}$ & $\begin{array}{c}\text { EU } \\
\hat{r}=0.61\end{array}$ & $\begin{aligned} \hat{R D} & \\
\hat{\rho} & =0.61 \\
\hat{\gamma} & =0.99\end{aligned}$ & $\begin{array}{c}\text { EU } \\
\hat{r}=0.82 \\
\hat{\alpha}= \\
-1.06\end{array}$ & $\begin{array}{c}\mathrm{RD} \\
\hat{r}=0.82 \\
\hat{\alpha}= \\
-1.06 \\
\hat{\gamma}=0.99\end{array}$ & $\begin{array}{c}\text { EU } \\
\hat{r}=0.53\end{array}$ & $\begin{array}{c}\mathrm{RD} \\
\hat{\rho}=0.53 \\
\hat{\gamma}=0.97\end{array}$ & $\begin{array}{c}\mathrm{EU} \\
\hat{r}=0.78 \\
\widehat{\alpha}= \\
-1.10\end{array}$ & $\begin{array}{c}\mathrm{RD} \\
\hat{r}=0.78 \\
\hat{\alpha}= \\
-1.10 \\
\hat{\gamma}=0.97\end{array}$ \\
\hline$\{77,0.5 ; 0\} \prec$ & 4.3 & 0.4 & 15.8 & 8.6 & & & & & & & & \\
\hline$\{30,0.5 ; 0\} \prec$ & 1.7 & 0.2 & 7.5 & 3.4 & 5.1 & 5.1 & 4.6 & 4.6 & & & & \\
\hline
\end{tabular}

${ }^{a}$ The higher payoff in a binary lottery is within the range of payoffs used in the experiment. Numbers are in U.S. dollars for the Holt-Laury and Harrison-Rutström studies and in British ponds for the Hey-Orme study in the middle columns.

${ }^{\mathrm{b}} \mathrm{p}$-values are larger than 0.1 